\title{
Implication of Margin of Glottic Cancer: Laser Surgery
}

\author{
Jae-Gu Cho \\ Jinho Jung \\ Min-Woo Park \\ Seung-Kuk Baek \\ Soon-Young Kwon \\ Kwang-Yoon Jung \\ Jeong-Soo Woo
}

Department of Otorhinolaryngology-Head and Neck Surgery, Korea University College of Medicine, Seoul, Korea
Received November 19, 2014

Revised December 9, 2014

Accepted December 12, 2014

\section{Correspondence}

Jae-Gu Cho

Department of Otorhinolaryngology-Head and Neck Surgery, Korea University College of

Medicine, 148, Gurodong-ro, Guro-gu,

Seoul 152-703, Korea

Tel: +82-2-2626-3189

Fax: +82-2-868-0475

E-mail: jgcholakorea.ac.kr

(c) Korean Society for Laser Medicine and Surgery

(c) This is an open access article distributed under the terms of the Creative Commons Attribution NonCommercial License (http://creativecommons.org/ licenses/by-nc/3.0) which permits unrestricted noncommercial use, distribution, and reproduction in any medium, provided the original work is properly cited.

\author{
Background and Objectives \\ An involved resection margin in a head and neck malignant neoplasm is \\ one of the most important factors affecting local recurrence. The aim of \\ this study is to assess the clinical impact of resected margin status in \\ laser surgery for glottic cancers on local recurrences and larynx \\ preservation.
}

\section{Materials and Methods}

Sixty four patients of glottis cancer from January 1994 to December 2013 were included in this study. The records were evaluated for disease recurrence rate, follow up duration, additional treatment modality, and laryngeal preservation. Pathologic reports with resection margin status were reviewed in all cases. Resection margins were evaluated in anterior, posterior, superior, inferior, and deep directions.

\section{Results}

Local recurrence was not associated with positive mucosal margin. The number of positive margins was also not associated with local recurrence ( $p=0.24)$. Only deep margin status showed association with local recurrence $(p<0.05)$. The laryngeal preservation rate was $97 \%$. All patients who underwent salvage laryngectomy had a positive deep margin.

\section{Conclusion}

Unlike other studies, in this study, mucosal margin did not have a significant impact on clinical outcome in laser surgery for early glottic cancer. However, a deep margin should be carefully evaluated during surgery and more study is needed.

\section{Key words}

Glottic cancer; Margin status; Laser surgery 


\section{INTRODUCTION}

Squamous cell cancer of the glottis is the most common laryngeal malignancy. Because of clear symptoms, sparse lymphohematologic drainage and solid anatomic boundaries, glottic cancer is commonly diagnosed in its early stage and is associated with an excellent cure rate. The goals of treatment of such lesions are cure of cancer with a low risk of complications, laryngeal preservation and good voice quality.

Despite the good prognosis of glottic cancer, repeated surgery even laryngectomy is needed for local recurrence. An involved resection margin in malignant neoplasms of the head and neck is one of the most important factors affecting local recurrence. ${ }^{1-4}$ However, the impact of margin status on the local recurrence of early glottic cancer remains controversial. Ansarin ${ }^{5}$ reported that patients with close or positive margins had greater recurrence risk. The few studies ${ }^{6-9}$ that have addressed the impact of margin status on local control in malignant vocal cord neoplasms have provided contrasting results.

The aim of this study is to assess the clinical impact of resection margin status in laser surgery for early glottic cancers on local recurrence and larynx preservation.

\section{MATERIALS AND METHODS}

This retrospective chart review and pathologic study was approved by the Ethics Committee and the Institutional Review Board at Korea University College of Medicine, Seoul, Korea.

From January 1994 to December 2013, the records of 64 consecutive patients confirmed to have squamous cell carcinoma of the glottis were identified (Table 1). The patients underwent laser surgery using a $\mathrm{CO}_{2}$ laser (Lumenis 30C, Yokneam, Israel) under general anesthesia. The $\mathrm{CO}_{2}$ laser was used in regular pulse continuous mode, spot size less than $3 \mathrm{~mm}$, with an output

Table 1. Patients and tumor characteristics

\begin{tabular}{lc}
\hline Characteristic & Value \\
\hline Age, median (range) & $59(32-77)$ \\
Sex (\%) & \\
Male & $58(84.4)$ \\
Female & $6(15.6)$ \\
Clinical stage & \\
Tis & 12 \\
T1a & 27 \\
T1b & 21 \\
T2 & 4 \\
\hline
\end{tabular}

power set at 1.5 to $3.5 \mathrm{~W}$.

Histological resection margin analysis was performed in all cases with the same technique and criteria. Surgical specimens were fixed in $4 \%$ formaldehyde for $72 \mathrm{hr}$, and inked on their superficial and deep mucosa with two different colored inks. The formaldehyde-fixed specimens were entirely embedded in paraffin. Sections $(4 \mu \mathrm{m})$ were mounted on glass slides and hematoxylin and eosin stained. Resection margins were analyzed in anterior, posterior, superior, inferior and deep directions. Positive margins were defined by in situ or invasive carcinoma in contact with the margin. All patients were followed-up with office-based fiberoptic or videostrobo-laryngoscopy monthly for the first 6 months and then every 2 months for the following 6 months. In the second year, patients were followed-up with office-based laryngoscopy every 3 to 4 months, and 4 to 6 months thereafter. Routine computed tomography and chest $\mathrm{X}$-rays were scheduled every year for at least 3 years post-operation. The records of consecutive patients were evaluated for disease recurrence rate, duration, additional treatment modality and laryngeal preservation.

Patient characteristics are presented as frequencies and percentages for categorical variables and as medians and ranges for continuous variables. The effect of margin status on recurrence rate was assessed using a multivariate Cox proportional hazards regression model. Statistical analysis was performed using SAS for Windows statistical software, version 8.2 (SAS Institute Inc., Cary, North Carolina, USA). A p value of less than 0.05 was considered to be statistically significant.

\section{RESULTS}

All laser surgery specimens were evaluated for surgical margin status by a pathologist. Margin status was analyzed in superior, inferior, anterior, posterior and deep directions. Surgical margins were positive in 47 patients. Local recurrence was observed in 17\% of patients with a positive margin but was not statistically significant. Of 47 patients, six were judged positive at the superior margin. Among these, one patient experienced local recurrence. Of 27 patients with a positive inferior margin, eight had local recurrence. Eight of 23 patients who were had a positive anterior margin experienced local recurrence. Local recurrence was not observed in patients with a positive posterior margin. Only deep margin status was associated with local recurrence and was statistically significant ( $p<0.05$ ) (Table 2). The superior margin positive population had the following values: $12.5 \%, 91.07 \%, 16.7 \%$ 
Table 2. Local recurrence according to margin status

\begin{tabular}{llccc}
\hline \multirow{2}{*}{ Margin Status } & \multicolumn{2}{c}{ Recurrence $(\mathrm{n}=8)$} & \multirow{2}{*}{ P value } \\
\cline { 3 - 4 } & & Positive & Negative & \\
\hline \multirow{2}{*}{ Superior } & Positive & 1 & 5 & 0.092 \\
& Negative & 7 & 51 & 0.342 \\
Inferior & Positive & 8 & 19 & 0.084 \\
& Negative & 0 & 37 & N/A \\
Anterior & Positive & 8 & 15 & 0.054 \\
& Negative & 0 & 41 & N/A \\
\multirow{3}{*}{ Posterior } & Positive & 0 & 8 & N/A \\
& Negative & 8 & 48 & 0.067 \\
& Positive & $6^{*}$ & 11 & 0.002 \\
& Negative & 2 & 45 & 0.405 \\
\hline
\end{tabular}

*Statistically significant.

and $87.9 \%$ for the sensitivity, specificity, PPV and NPV, respectively. The inferior and anterior margin positive populations had similar results. However, the deep margin positive population had the following results: $75 \%$, $80.4 \%, 35.3 \%$ and $95.7 \%$ for the sensitivity, specificity, PPV and NPV, respectively. The sensitivity and NPV in the deep margin positive population are higher than those of the superior margin positive population. The sensitivity and NPV in the inferior and anterior margin positive populations are higher than those of the deep margin positive population, but their specificity and PPV are lower than those of the deep margin positive population.

\section{DISCUSSION}

Laser resection of glottic cancer was developed in the $1970 \mathrm{~s} .{ }^{10}$ It is based on the same principle of en bloc resection as partial laryngectomy, but laser surgery shortens hospital stay and simplifies postoperative course, notably avoiding tracheotomy. ${ }^{11}$ Since the initial reports from the 1970s, laser surgery has evolved to be a widely accepted operation in the treatment of early laryngeal carcinoma. Currently, laser surgery is recognized as an effective and safe standard management option for patients with early glottic cancer. When performing laser surgery to treat early glottic cancer, resection with a narrow tumor margin is usually the goal in order to preserve as much tissue as possible. The peculiarities of laryngeal embryologic development and the scarce glottic submucosal lymphatic network suggest that the resection margins can be narrower than required in other head and neck cancers.

The present study sought to assess the margin status in terms of local recurrence in laser surgery for early glottic cancer. In our results, there is a discrepancy between pathology reports with a high percentage of unclear resection margins and a low incidence of recurrence. Forty-seven patients with a positive margin were either violated or showed tumor-suspect areas in the laser carbonized zone with thermal artifact. In this group, only eight patients experienced recurrence; however, no instances of recurrence were observed when the surgical margins were negative on permanent pathologic reports although this was not statistically significant. This finding suggests that mucosal margins do not have a significant influence on clinical outcomes. We assumed that there were no tumors in the resection margin even if positive margins were revealed in the pathologic report or there were burns and artifacts in the margin which did not allow an exact conclusion. Deep margin status, however, was associated with local recurrence. Six of 17 patients who were judged positive at the deep margin experienced local recurrence $(p<0.05)$. The high rate of local recurrence observed in this group could be explained by conservative resection of the lesion, which was due to an attempt to save the vocalis muscle and achieve good phonatory results. The other reason was that the deep plane of excision was the most difficult part of the operation, especially if it was uncertain whether the vocal ligament was affected or not. On the other hand, the superior, inferior, anterior and posterior margins were not associated with local recurrence.

Laser excision of early glottic cancer has several advantages over other treatment modalities. The procedure is brief with shorter hospital stay, lower morbidity, and fewer side effects. In addition, organ preservation is the best advantage. In this study, the organ preservation rate was $97 \%$. The local control rate with laryngeal preservation was similar to that seen in other studies. But two patients who had positive deep margin required salvage total laryngectomy after radiation therapy. Even though our data supported that deep margin should be carefully evaluated during operation, this study was primarily limited by the number of $\mathrm{T} 2$ patients. There might be some other limiting factors, such as histologic characteristics and laser cordectomy type, which were not classified nor taken into consideration in the analysis, although they could also potentially affect local recurrence. Finally, the retrospective nature of the results was a limitation.

The margin status in early glottic cancer was important for local recurrence. But mucosal margin did not have a significant impact on clinical outcome unlike other studies. Only positive deep margin was significantly 
correlated with local recurrence and larynx preservation. Deep margin should be carefully evaluated during operation and the more study was needed.

\section{REFERENCES}

1. Looser KG, Shah JP, Strong EW. The significance of "positive" margins in surgically resected epidermoid carcinomas. Head Neck Surg 1978;1:107-11.

2. Loree TR, Strong EW. Significance of positive margins in oral cavity squamous carcinoma. Am J Surg 1990;160:410-4.

3. Byers RM, Bland KI, Borlase B, Luna M. The prognostic and therapeutic value of frozen section determinations in the surgical treatment of squamous carcinoma of the head and neck. Am J Surg 1978;136:525-8.

4. Ho CM, Ng WF, Lam KH, Wei WI, Yuen AP. Radial clearance in resection of hypopharyngeal cancer: an independent prognostic factor. Head Neck 2002;24:181-90.

5. Ansarin M, Santoro L, Cattaneo A, Massaro MA, Calabrese L, Giugliano $G$, et al. Laser surgery for early glottic cancer: impact of margin status on local control and organ preservation. Arch Otolaryngol Head Neck Surg 2009;135:385-90.
6. Hartl DM, de Monès E, Hans S, Janot F, Brasnu D. Treatment of early-stage glottic cancer by transoral laser resection. Ann Otol Rhinol Laryngol 2007;116:832-6.

7. Sigston E, de Mones E, Babin E, Hans S, Hartl DM, Clement $P$, et al. Early-stage glottic cancer: oncological results and margins in laser cordectomy. Arch Otolaryngol Head Neck Surg 2006;132:147-52.

8. Crespo AN, Chone CT, Gripp FM, Spina AL, Altemani A. Role of margin status in recurrence after CO2 laser endoscopic resection of early glottic cancer. Acta Otolaryngol 2006;126:30610.

9. Jäckel MC, Ambrosch P, Martin A, Steiner W. Impact of reresection for inadequate margins on the prognosis of upper aerodigestive tract cancer treated by laser microsurgery. Laryngoscope 2007;117:350-6.

10. Strong MS, Jako GJ. Laser surgery in the larynx. Early clinical experience with continuous CO 2 laser. Ann Otol Rhinol Laryngol 1972:81:791-8.

11. Gallo A, de Vincentiis M, Manciocco V, Simonelli M, Fiorella ML, Shah JP. CO2 laser cordectomy for early-stage glottic carcinoma: a long-term follow-up of 156 cases. Laryngoscope 2002;112:370-4 